\title{
Periodic Solutions of Certain Fifth Order Differential Equations
}

\author{
Melike Karta \\ Department of Mathematics, Faculty of Science and Arts, \\ Agri Ibrahim Cecen University, Agri-Turkey \\ E-mail: melike_karta2010@hotmail.com
}

\begin{abstract}
In this study, it is investigated that no periodic solution other than the trivial solution $X=0$ to the nonlinear fifth order differential equation$$
X^{5}+A X^{4}+B \dddot{X}+G(X) \dot{X}+\Theta\left(X, \dot{X}, \ddot{X}, \dddot{X}, X^{(4)}\right) \ddot{X}+F(X, Y, Z, W, U) X=0
$$

by Lyapunov function.
\end{abstract}

Keywords: Nonlinear vector differential equation of fifth, Lyapunov function, Periodic solutions

DOI: $10.7176 / \mathrm{JSTR} / 5-2-01$

\section{Introduction}

Periodic solutions of high order scalar and vector differential equations have appeared in the sciences as some practical mechanical problems, physics, chemistry, biology, economics, and control theory. According to studies avaliable in the literature, the problems related to the periodic behavior of solutions of a higher order non-linear scalar and vector differential equation have been analyzed by many authors. Bereketoğlu [2-3] have obtained periodic solutions of certain class of seventh-order and eighth-order differential equations. Ezeilo [4-6] have proposed periodic solutions of a certain fourth and fifth order differential equations. Tejumola [8] have considered instability and periodic solutions of certain nonlinear seventh-order and sixth-order ordinary differential equations. Tiryaki [9] have analyzed periodic solutions of a certain fourth and fifth order differential equations. Tunç [10] have presented periodic solutions of a certain fourth and fifth order vector differential equations.In all of the papers mentioned above, authors used Lyapunov's second (or direct) method [11].

LI and $\mathrm{Yu}[1]$ investigated the instability of trivial solution to fifth-order nonlinear scalar differential equation

$$
x^{(5)}+a x^{(4)}+b \dddot{x}+\varphi\left(x, \dot{x}, \ddot{x}, \dddot{x}, x^{(4)}\right) \ddot{x}+g(x) \dot{x}+f(x)=0
$$

by introducting a Lyapunov function, where $\mathrm{a}$ and $\mathrm{b}$ are some positive constants.

Karta [7] examined the instability of trivial solution of the fifth order non-linear vector differential equation

$X^{(5)}+A X^{(4)}+B \dddot{X}+\Theta\left(X, \dot{X}, \ddot{X}, \dddot{X}, X^{(4)}\right) \ddot{X}+G(X) \dot{X}+F(X, Y, Z, W, U) X=0$

In this study, we are concerned with periodic solution of the solution $X=0$ of the fifth order nonlinear vector differential equation described by (1.1). In the real Euclidean space $R^{n}$, where $X \in R^{n} ; A$ and $B$ are constant $n x n$-symmetric matrices; $\Psi, G$ and $F$ are $n x n$-symmetric continuous matrix function. We use, 
instead of (1.1) the equivalent differential system

$$
\begin{gathered}
\dot{X}=Y, \dot{Y}=Z, \dot{Z}=W, \dot{W}=U \\
\dot{U}=-A U-B W-\Psi(X, Y, Z, W, U) Z-G(X) Y-F(X, Y, Z, W, U) X
\end{gathered}
$$

which was obtained as usual by setting

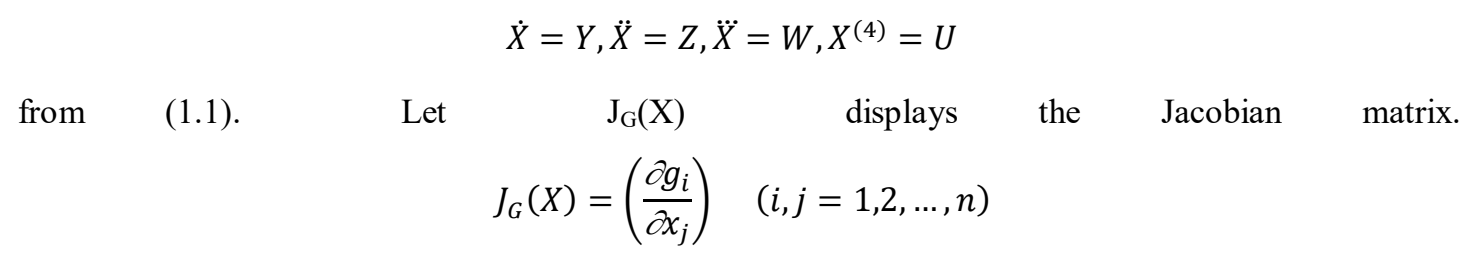

in which $\left(x_{1}, x_{2}, \ldots, x_{n}\right)$ and $\left(g_{1}, g_{2}, \ldots, g_{n}\right)$ are components of $\mathrm{X}$ and G, respectively.

\section{Main results}

Our main result is the following the theorem.

\section{Theorem 2.1.}

In addition to the basic assumptions imposed on $A, B, \Psi, G$ and $F$ appearing in (1.1), we assume there are constants $\mathrm{a}, \mathrm{b}$ and a positive constant $\mathrm{k}$ such that the following conditions hold

(i) $\quad \lambda_{i}(A) \geq a, \lambda_{i}(A) \geq b$, bsgna $>0$

(ii) $\left.\quad \lambda_{i}(F(X)) \operatorname{sgna}-(1 / 4|a|) \lambda_{i}(\Psi(X, Y, Z, W, U))\right)^{\wedge} 2 \geq k(i=1,2, \ldots, n)$

Then equation (1.1) has no periodic solution other than $\mathrm{X}=0$.

Proof. Let $\left(\varphi_{1}, \varphi_{2}, \varphi_{3}, \varphi_{4}, \varphi_{5}\right)=\left(\varphi_{1}(t), \varphi_{2}(t), \varphi_{3}(t), \varphi_{4}(t), \varphi_{5}(t)\right)$

be an arbitraray $\alpha$-periodic solution of $(1.1)$, that is

$$
\begin{aligned}
\left(\varphi_{1}(t), \varphi_{2}(t), \varphi_{3}(t), \varphi_{4}(t), \varphi_{5}(t)\right) \\
\quad=\left(\varphi_{1}(t+\alpha), \varphi_{2}(t+\alpha), \varphi_{3}(t+\alpha), \varphi_{4}(t+\alpha), \varphi_{5}(t+\alpha)\right)
\end{aligned}
$$

For some $\alpha>0$. It will be shown that, under the conditions in Theorem (2.1),

$$
\varphi_{1}=\varphi_{2}=\varphi_{3}=\varphi_{4}=\varphi_{5}=0 .
$$

As basic tool the proof of Theorem (2.1), we will use Lyapunov function

$$
V=V_{0}(X, Y, Z, W, U) \text { sgna }
$$

given as 


$$
\begin{gathered}
V_{0}=\langle Y, W\rangle+\langle Y, A Z\rangle-\langle X, U\rangle-\langle X, A W\rangle-\langle X, B Z\rangle-\frac{1}{2}\langle Z, Z\rangle+\frac{1}{2}\langle B Y, Y\rangle-\int_{0}^{1}\langle\sigma G(\sigma X) X, X\rangle d \sigma \\
\theta(\mathrm{t})=V_{0}\left(\varphi_{1}(t), \varphi_{2}(t), \varphi_{3}(t), \varphi_{4}(t), \varphi_{5}(t)\right) \text { sgna. }
\end{gathered}
$$

Since $\theta(\mathrm{t})$ is continuous and $\varphi_{1}, \varphi_{2}, \varphi_{3}, \varphi_{4}, \varphi_{5}$ are periodic in $t, \theta(\mathrm{t})$ is clearly bounded. An elementary differentiation will Show that

$$
\dot{V}_{0}=\frac{d}{d t} V_{0}(X, Y, Z, W, U)=\langle A Z, Z\rangle+\langle F(X) X, X\rangle+\langle X, \Psi(X, Y, Z, W, U) Z\rangle
$$

and it is clear $\dot{V} \geq k\|X\|^{2} \geq 0$ that, under the conditions in Theorem (2.1).

Hence $\dot{\theta(t)} \geq 0$; so that $\theta(\mathrm{t})$ is monotone in $t$, and therefore, being bounded, tends to a limit, $\theta_{0}$ say, as $t \rightarrow+\infty$. That is $\lim _{t \rightarrow+\infty} \theta(\mathrm{t})=\theta_{0}$. It is readily showed that

$$
\theta(\mathrm{t})=\theta_{0} \text { for all } t
$$

from by (2.1),

$$
\theta(t)=\theta(t+m \alpha)
$$

For any arbitrary fixed $t$ an for arbitrary integer $m$, and then for $t \rightarrow+\infty$, it implies that

$$
\lim _{t \rightarrow+\infty} \theta(\mathrm{t})=\lim _{t \rightarrow+\infty} \theta(\mathrm{t}+\mathrm{m} \alpha)=\theta_{0}
$$

Due to $\lim _{t \rightarrow+\infty} \theta(\mathrm{t})=\theta_{0}$ and $\theta(\mathrm{t})=\theta(\mathrm{t}+\mathrm{m} \alpha)$ for all $t$, it is $\theta(\mathrm{t})=\theta_{0}$.thus for all $t \dot{\theta}(t)=0$. Furthermore $\dot{\theta}(t)=0$ necessarily implies that $\varphi_{1}=0$ for all $t$. That is, it is showed that

$$
\varphi_{1}=\varphi_{2}=\varphi_{3}=\varphi_{4}=\varphi_{5}=0
$$

Because $\varphi_{1}, \varphi_{2}, \varphi_{3}, \varphi_{4}, \varphi_{5}$ are solutions of (1.2) system, it is obtained

$$
\left(\varphi_{1}(t), \varphi_{2}(t), \varphi_{3}(t), \varphi_{4}(t), \varphi_{5}(t)\right)=(X(t), Y(t), Z(t), W(t), U(t))=(0,0,0,0,0)
$$

This completes the proof of Theorem (2.1).

\section{Conclusion}

The proof of the Theorem 2.1 showed that the equation (1.1) have no periodic solution other than the trivial solution $X=0$. So, this has helped us to find a new result.

\section{References}

[1] Bereketoğlu, H., (1992). On the periodic solutions of certain class of seventh-order differential equations. Periodica Mathematica Hungarica, H. 24, 13-22.

[2] Bereketoğlu, H., (1992), On the periodic solutions of certain class of eighth-order differential 
equations. Commun. Fac. Sci. Univ.Ank.Series A, H. 41, 55-65.

[3] Ezeilo, J.O.C., (1979). Periodic solutions of a certain fourth order diffrential equations. Atti. Accad. Naz. Lincei CI. Sci. Fis. Mat. Natur, LXVI, 344-350.

[4] Ezeilo, J.O.C., (1979). A further instability theorem for a certain fifth -order diffrential equations. Math.proc.cambridge Philos. Soc., 86,491-493.

[4] Ezeilo, J.O.C., (1983). Uniqueness theorems for periodic solutions of certain fourth and fifth order diffrential systems. Journal of the Nigerian mathematical society, 2, 55-59.

[5] Karta, M., (2015), further instability result on the oltions of nonlinear vector differential equations of the fifth order. Eastern Anatolian journal of science, vol.1, 29-34.

[6] LI, W.J., and YU, Y.H. (1990), Instability theorems for some fourth-order and fifth-order differential equations.(Chinese) J.Xiniang Univ. Natur. Sci, 7, 7-10.

[7] Lyapunov, A.M., (1966). Stability of Motion. Academic Press,New York -London, 203p

[8] Tejumola, H.O., (2000). Instability and perodic solutions of certain nonlinear diffrential equations of orders six and seven, in: Ordinary diffrential equations.(Abuja, 2000). Proc.natl. Math. Cent. Abuja Niger, 1.1, Natl. Math. Cent, (2000), 55-65.

[9] Tiryaki, A., (1990). On the periodic solutions of certain fourth and fifth order differential equations. Pure and Applied Mathematica Sciences, XXXII, 1-2.

[10] Tunç, E ., .,(2005) On the periodic solutions of certain fourth and fifth order vector differential equations . Mathematical communicatıons, 10,135-141. 